\title{
Differences in Norwegian and Swedish student teachers' explanations of solutions of linear equations
}

\author{
Niclas Larson ${ }^{1}$, and Kerstin Larsson ${ }^{2},{ }^{1}$ University of Agder, and ${ }^{2}$ Stockholm University
}

\begin{abstract}
This study draws on data from 146 Norwegian and 161 Swedish student teachers. They were given a correct but short and unannotated solution to the linear equation $x+5=4 x-1$. The student teachers were invited to explain the solution provided for a fictive friend, who was absent when the teacher introduced this topic. An accurate solution of this equation contains two additive and one multiplicative operation.
\end{abstract}

There are two main strategies for solving a linear equation, 'swap sides swap signs' (SSSS) and 'do the same to both sides' (DSBS). Of the Norwegian student teachers, 2/3 explained the additive steps in the solution by SSSS, while only $1 / 3$ of the Swedish student teachers applied SSSS. Consequently, DSBS was more frequent among the Swedish student teachers regarding the additive steps. However, in the final, multiplicative step, 3/4 of the Norwegian student teachers chose to explain by DSBS. On the contrary, among the Swedish student teachers the proportion applying DSBS for the multiplicative step of the solution decreased, and almost as many provided a deficient explanation of the final operation.

We discuss possible reasons for differences between the nations. We also suggest how teacher educators in both countries can use the results of this study to improve student teachers' explanations of how to solve linear equations.

\section{INTRODUCTION}

The topic of linear equations is a part of school mathematics all over the world (Andrews \& Sayers, 2012). In the Norwegian and Swedish curricula, the concept of equation is first mentioned for middle school, that is pupils between the age of 9 and 13 (Norwegian Directorate for Education and Training, 2020; Swedish National Agency for Education, 2019). However, the notion of equalities is mentioned already in lower primary school. Thus, knowledge of teaching how to solve equations is relevant for all student teachers in mathematics.

Linear equations with a single unknown can roughly be divided into equations with the unknown on one side of the equal sign only, and equations with unknown terms on both sides (Andrews, 2020). While the former can be solved by inverse arithmetic operations or informal techniques as 'cover the unknown', the latter tend to require a more structured approach. Hence, explaining the procedure of solving equations is essential for student teachers.

This paper draws on data from a task used in previous studies on student teachers in Cyprus (Andrews \& Xenofontos, 2017) and upper secondary school students in Sweden (Andrews \& Öhman, 2019). The participants were presented a correct solution of an equation, but with no annotations that indicated the hidden solver's thinking. The four lines in the solution were:

$x+5=4 x-1 \quad(1) ; \quad 5=3 x-1(2) ; \quad 6=3 x \quad(3) ; \quad 2=x$ (4)

The participants were then invited to explain how the equation was solved, to a friend who was absent when the topic was introduced by the teacher.

Andrews and Xenofontos' (2017) framework for analysing the replies was refined by Andrews and Larson (2019) to a set of 13 low-inference codes. Seven of these codes deal with two fundamental principles for solving equations, 'swap sides swap signs' (SSSS) and 'do the same to both sides' (DSBS). In the current equation, the operations from line (1) to (2) and (2) to (3) are additive, while the operation from (3) to (4) is multiplicative. A typical explanation of the step from (2) to (3) by SSSS would be 'we move -1 to the left-hand side, and then minus becomes plus', while DSBS could include 'to get rid of -1 , we add 1 to both sides'. Although both principles entirely work for solving linear equations, DSBS 
more clearly illuminates the steps of the solution, while SSSS rather masks the mathematical understanding (Andrews, 2020).

Previous investigations showed that a vast majority of student teachers on Cyprus explained the additive steps by SSSS (Andrews \& Xenofontos, 2017), while the Swedish upper secondary school students tended to use DSBS (Andrews \& Öhman, 2019). Prompted by the interviewer, Swedish upper secondary students showed awareness of SSSS too, but also commented that using SSSS makes you not understand what you are doing, and that their teacher not allowed them to use SSSS. Despite no definite conclusions can be drawn from two studies, this still may arouse an awareness of the cultural influence on teaching (cf. Andrews \& Larson, 2017).

This paper draws on data from one university in Norway and one in Sweden (see Andrews, 2020, for a report on the Swedish data). The participants were student teachers following their first course in mathematics, and the data collection was made before the topic of equations was treated in the course. Collecting data in two countries enables us to make comparisons between the countries. Our research question is "How do Norwegian and Swedish student teachers explain how to solve a linear equation?"

\section{METHOD}

The Norwegian participants $(N=146)$ followed a programme for primary or lower secondary schoolteachers $(1-7$ or $5-10)$, while the Swedish $(N=161)$ followed the teacher education for primary school (F-3 or 4-6). The task was presented on an A4-sheet, where the lower half was blank. Data were collected anonymously. The student teachers' participation was voluntary. All student teachers fulfilling the task agreed to participate in the study.

Andrews and Larson's (2019) framework with 13 low-inference codes contained two codes about SSSS and five codes about DSBS. The DSBS codes were named "DSBS general", "DSBS general additive", "DSBS particular additive", "DSBS general multiplicative" and "DSBS particular multiplicative". Here, general was used for scripts explaining the principle without referring to the equation given in the task, while particular meant the explanation referred to the current equation. This means 'you can add or subtract both sides by any number' would be "DSBS general additive", while 'for $6=3 x$, you divide both sides by 3' would be "DSBS particular multiplicative". For SSSS, only the two codes for "general" and "particular additive" were included in this set of 13 codes. However, to create a 'symmetric' set of codes, the remaining three codes were added for SSSS, despite the two multiplicative codes in practice would be empty, and that general almost always refers to the additive aspect of "SSSS general". Hence, the framework applied for this study consisted of 16 codes.

Besides the 10 codes presented, also the code "Unspecified operation on the coefficient" deals with the operational steps of the solution. An example of this code is to explain the step from (3) to (4) by "to get the value of $x$, you divide 6 by 3', without pointing out that you divide both sides by 3 . Five codes remain. "Discusses the nature of $x$ " means the student in some way expressed that $x$ is or represents a variable or an unknown. "Equality of both sides" deals with keeping the balance of both sides of the equal sign. "Conceptual objective" means the student pointed out that the goal was to find the value of $x$, while "Procedural objective" described the purpose of the operations, for example to separate knowns and unknowns to different sides of the equal sign. "Checks solution" is self-explanatory. (See Andrews \& Larson, 2019, for an accurate presentation of all codes.)

The first author coded the Norwegian scripts, while the second author coded the Swedish. To check for consistence between the authors, 20 scripts from each stack were chosen by random to be coded by the other author. Calculations gave Cohen's kappa 0.82, which shows the consistency to be of high quality. The main inconsistencies appeared between "SSSS general" and "SSSS general additive", which, as expected, were difficult to distinguish. Aggregating these variables, which basically not affected the outcome of the coding, resulted in a Cohen's kappa of 0.89 , which is excellent. The final coding was used for the statistical analyses that yielded our results.

\section{RESULTS}

We start by presenting the four codes that identifies the objectives of solving an equation. Table 1 shows there were no clear differences between the countries regarding discussing the nature of $x$, or the equality of both sides in the equation. In addition, these two codes were not very frequent (between $12 \%$ and 
$20 \%$ of the student teachers). The other two codes, "Conceptual objective" and "Procedural objective", were more frequent among the Norwegian student teachers.

Table 1. Frequencies of the four codes that identify the objectives of solving an equation.

\begin{tabular}{|l|cc|cc|cc|cc|}
\cline { 2 - 9 } \multicolumn{1}{c|}{} & \multicolumn{2}{|c|}{$\begin{array}{c}\text { Discusses the } \\
\text { nature of } x\end{array}$} & \multicolumn{2}{|c|}{$\begin{array}{c}\text { Equality of both } \\
\text { sides }\end{array}$} & \multicolumn{2}{|c|}{$\begin{array}{c}\text { Conceptual } \\
\text { objective }\end{array}$} & \multicolumn{2}{|c|}{$\begin{array}{c}\text { Procedural } \\
\text { objective }\end{array}$} \\
\cline { 2 - 9 } & Yes & No & Yes & No & Yes & No & Yes & No \\
\hline Norway $(N=146)$ & 22 & 124 & 24 & 122 & 65 & 81 & 128 & 18 \\
\hline Sweden $(N=161)$ & 20 & 141 & 33 & 128 & 47 & 114 & 95 & 66 \\
\hline
\end{tabular}

Regarding explanations of the operations in the solution of the equation, some clear differences between the countries were identified in the student teachers' utilisation of SSSS or DSBS. Table 2 presents the five codes for which the explanation included reference to the equation given in the task.

Table 2. Frequencies of five of the codes identified in the explanations of the operational steps of the solution.

\begin{tabular}{|c|cc|cc|cc|cc|cc|}
\cline { 2 - 11 } \multicolumn{1}{c|}{} & $\begin{array}{c}\text { SSSS particular } \\
\text { additive }\end{array}$ & \multicolumn{2}{|c|}{$\begin{array}{c}\text { SSSS particular } \\
\text { multiplicative }\end{array}$} & \multicolumn{2}{|c|}{$\begin{array}{c}\text { DSBS particular } \\
\text { additive }\end{array}$} & $\begin{array}{c}\text { DSBS particular } \\
\text { multiplicative }\end{array}$ & $\begin{array}{c}\text { Unspecified operation } \\
\text { on the coefficient }\end{array}$ \\
\cline { 2 - 12 } & Yes & No & Yes & No & Yes & No & Yes & No & Yes & No \\
\hline Norway $(N=146)$ & 94 & 52 & 1 & 145 & 31 & 115 & 111 & 35 & 11 & 135 \\
\hline Sweden $(N=161)$ & 57 & 104 & 8 & 153 & 72 & 89 & 56 & 105 & 51 & 110 \\
\hline
\end{tabular}

To explain the additive steps by SSSS was more frequent among the Norwegian student teachers, where about $2 / 3$ applied SSSS. In addition, 60 of these 94 Norwegian student teachers also provided some general explanation of the SSSS principle, not referring explicitly to the current equation. The Swedish proportion applying "SSSS additive" was $1 / 3$, and only 10 of the 57 Swedish student teachers provided general aspects of SSSS. However, the Swedish student teachers were much more frequent in utilising DSBS to explain the additive steps of the solution than the Norwegian student teachers were.

Although preferring SSSS in the additive steps, $3 / 4$ of the Norwegian student teachers chose to explain the final, multiplicative step by DSBS. Among the Swedish student teachers, the use of DSBS decreased for the multiplicative step, and almost the same number gave an explanation coded as "Unspecified operation on the coefficient", a code almost totally absent among the Norwegian student teachers.

We close this section by mentioning the last code, which is "Checks solution". This code was not frequent, only 13 of the 146 Norwegian student teachers and 8 of the 161 Swedish student teachers commented on or showed that the solution can be checked.

\section{DISCUSSION}

The Norwegian student teachers included explanations coded as "Conceptual objective" and "Procedural objective" to a greater extent than the Swedish (see Table 1). The data do not reveal the reasons, but the result is anyway important. It shows that teacher educators in both countries better can emphasise the importance of objectives in explanations of how to solve equations. That is important especially for Swedish student teachers. Teacher educators should also highlight as well that the goal is to find the value of the unknown (conceptual objective), as that a key to the solution is to get the unknown alone on one side of the equal sign (procedural objective). Since the conceptual objective was the least frequent among all teacher students, it is of special importance to stress that part. Possibly that will improve future teachers' ways of explaining how to solve equations.

The results in Table 2 are noteworthy, that is how the student teachers described the operational steps in the solution of the equation. The Swedish student teachers preferred to explain the additive steps by DSBS rather than by SSSS, even though the preference of DSBS was moderate. For the Norwegian 
student teachers, the results were the opposite. They preferred SSSS for the additive steps, and in specific just a small proportion chose to explain by DSBS. However, when it came to the final step, to go from $6=3 x$ to $2=x$, a clear majority of the Norwegian student teachers chose DSBS, and very few provided inadequate explanations as 'you divide 6 by 3', which was more common among the Swedish student teachers. A relevant question is why student teachers, who in the additive step avoided DSBS, switched to DSBS for the multiplicative step. Another relevant question is why the Swedish student teachers rather went the other way, that is the proportion applying DSBS was lower for the multiplicative step.

It is fair to conclude that cultural influences play a role for student teachers' choices of either SSSS or DSBS in the additive steps. The participants were student teachers in their first mathematics course in the teacher education, so it is likely that their understanding of solving equations was rooted in their upper secondary education. Cultural influences are important for teaching (Andrews \& Larson, 2017), and stressing DSBS might be more frequent among Swedish upper secondary teachers than among their Norwegian colleagues. As Andrews and Öhman (2017) described, their interviewees claimed their teacher did not allow them to use SSSS. One upper secondary student's statement, that when applying SSSS you will not understand what you are doing, is possibly connected to the Swedish teaching culture. Furthermore, that DSBS elucidates the principles of equation solving, while SSSS masks them (Andrews, 2020), supports this statement. This does not mean providing SSSS is wrong, but to understand what you are doing, SSSS should be backed up by DSBS (Andrews \& Öhman, 2017). Although there might be cultural traditions that stress a specific method (Andrews \& Larson, 2017), teacher educators must also raise awareness of its shortcomings.

It is not obvious how to apply "SSSS multiplicative", because 'swapping signs' naturally refers to changing plus for minus or vice versa. Swapping sides in a multiplicative step would rather refer to swap to the inverse operation than to swapping signs. This might be one explanation of why so many of the Norwegian student teachers chose to change their approach to DSBS in the final division. SSSS was not applied in the final step by the Swedish student teachers either, but almost one third of them did not point out that you must divide both sides by 3 . One hypothesis is that they saw the final step as so trivial that they did not find it worth explaining. The equation $6=3 x$ can easily be solved by informal methods (cf. Andrews, 2020), so emphasising that you must divide both sides by 3 might have been considered as superfluous.

Choosing one method for the additive step, does not imply you do not understand the other. It might be just that you prefer one of the methods, possibly by cultural reasons (Andrews \& Larson, 2017). In this study, the participants gave a written reply, with no possibilities to follow-up questions. It is not unlikely that several student teachers would have given an alternative method, or a justification of the SSSS additive method, if prompted (Andrews \& Öhman, 2017). A few participants included such a justification anyway, by explaining that the consequence of DSBS is that the term appears on the other side with the opposite sign. Despite just a few student teachers included this justification, it is plausible that more student teachers are aware of it. Correspondingly, the Swedish student teachers who provided explanations coded as "Unspecified operation on the coefficient" might give DSBS as an alternative, if prompted. However, from our data, it is not possible to draw further conclusions about that.

The use of low-inference codes is a rather rough way to analyse data. If a script is marked "Procedural objective", this explanation might still be of low quality. In a good explanation, the objectives should come early, to explain the purpose of the solution before the operations are explained. If the objective appears only in the last step, like 'to get $x$ alone we divide both sides by 3', the idea behind the solution might not be clear during the process. Yet, low-inference codes reveal important aspects, and may also be a starting point for qualitative analyses. This is, however, beyond the scoop of this paper.

\section{CONCLUSIONS}

Despite this study was based on data from one university in each country only, it was possible to draw some conclusions. We summarise by highlighting three issues, to be emphasised by teacher educators. First, it is important to early clarify the objectives of solving an equation. This should particularly be stressed for Swedish student teachers. Second, to mainly be stressed in Norway, all student teachers should provide a DSBS explanation of the additive steps. Third, Swedish student teachers must explain the final division more clearly, even in cases where the result is obvious. 
Of course, a teacher educator may want to emphasise the nature of $x$, the balance property in an equation, and the possibility of checking your solution too. Nevertheless, the three issues mentioned above might be a good starting point for the teaching towards improvement of student teachers' explanations of how to solve equations.

\section{ACKNOWLEDGMENTS}

The authors are grateful to Paul Andrews for his contribution by collecting the Swedish data.

\section{REFERENCES}

Andrews, P. (2020). Swedish primary teacher education students' perspectives on linear equations. Nordic Studies in Mathematics Education, Vol. 25, No. 2, pp. 29-48.

Andrews, P, \& Larson, N. (2017). Swedish upper secondary students' perspectives on the typical mathematics lesson. Acta Didactica Napocensia, Vol. 10, No. 3, pp. 109-121.

Andrews, P., \& Larson, N. (2019). The development of a set of low-inference codes for uncovering students' understanding of linear equations: Facilitating comparative analysis. In L. Harbison \& A. Twohill (Eds.) Proceedings of the Seventh Conference on Research in Mathematics Education in Ireland (pp. 35-42). Institute of Education, Dublin City University.

Andrews, P., \& Öhman, S. (2017). Don't 'swap the side and swap the sign', you won't understand what you are doing: Swedish students' perspectives on linear equations. Paper presented at The Eighth Nordic Conference on Mathematics Education, Stockholm University, 30 May - 2 June 2017.

Andrews, P., \& Sayers, J. (2012). Teaching linear equations: Case studies from Finland, Flanders and Hungary. The Journal of Mathematical Behavior, Vol. 31, No. 4, pp. 476-488.

Andrews, P., \& Xenofontos, C. (2017). Beginning teachers' perspectives on linear equations: A pilot quantitative comparison of Greek and Cypriot students. In T. Dooley \& G. Gueudet (Eds.), Proceedings of the Tenth Congress of the European Society for Research in Mathematics Education (pp. 1594-1601). Institute of Education, Dublin City University.

Norwegian Directorate for Education and Training. (2020). Curriculum for the common core subject of mathematics (MAT1-05). Norwegian Directorate for Education and Training.

Swedish National Agency for Education. (2019). Curriculum for the compulsory school, preschool class and school-age educare. Swedish National Agency for Education. 